\title{
United States Immunodeficiency Network
}

National Cancer Institute

\section{Source}

National Cancer Institute. United States Immunodeficiency Network. NCI Thesaurus.

Code C88717.

A research consortium established to advance scientific research in the primary immune deficiency diseases (PIDD). 